\title{
Double sero-negative myasthenia gravis associated with thymic hyperplasia and autoimmune thyroiditis in a young patient
}

\author{
Maria-Gabriela Catana ${ }^{1}$, Liliana Cristina Cuibus ${ }^{1}$, Andreea Dan ${ }^{1}$, \\ Corina Roman-Filip ${ }^{1,2}$ \\ ${ }^{1}$ Department of Neurology, Emergency Clinical Hospital, Sibiu, Romania \\ 2"Lucian Blaga" University, Sibiu, Romania
}

\begin{abstract}
Myasthenia gravis is an autoimmune disorder affecting the post-synaptic neuromuscular junction. Clinically it evolves with fluctuating muscle weakness. In $13-22 \%$ of the reported cases, another autoimmune disorder is associated. We report the case of a 21 years old woman presenting at 4 months after deliverance, global muscle weakness, dysphonia, dysarthria and dysphagia. Biological tests reveal: AchR antibodies and anti-MUSK antibodies - negative, TSH 4,68 $\mu \mathrm{lU} / \mathrm{ml}$ (normal range: 0,4-4 $\mu \mathrm{lU} / \mathrm{ml}$ ), FT4 0,785 ng/dl (normal range: 0,89-1,76 ng/dl), anti thyroglobulin antibod-ies 183,01 IU/mL (normal range: $<4,11 \mathrm{IU} / \mathrm{mL}$ ), Chest CT - thymic hyperplasia and EMG - decremental response at repetitive stimulation. It is established the diagnosis: myasthenia gravis Osserman stage $\mathrm{Ilb}$. After treatment with acetylcholine esterase inhibitors and corticosteroids the patient's symptomatology has improved.
\end{abstract}

Keywords: myasthenia gravis, double sero-negative, antibodies, autoimmune, thymic hyperplasia

\begin{abstract}
Abrevieri
MG - Myasthenia Gravis

dSNMG - double Sero-Negative Myasthenia Gravis

AchR - acetylcholine

MUSK - muscle specific receptor tyrosine kinase
\end{abstract}

\section{INTRODUCTION}

Myasthenia gravis is an antibody mediated autoimmune disorder of the neuromuscular junction. In most cases, it is caused by pathogenic autoantibodies directed towards the skeletal muscle acetylcholine receptor (AchR - in $80 \%$ of the cases reported of generalized $\mathrm{MG}$ ) or against components of postsynaptic muscle endplate, such as muscle specific receptor tyrosine kinase (MUSK) (5\% of patients with generalized MG) $(2,7)$. It is also believed that the hyperplastic thymus gland is involved in the initiation of the immune response and the production of antibodies, especially AchR antibodies (2). Recent studies have shown that serial antibody titers tend to correlate with disease status. It was found a correlation between anti-AChR antibody levels and maximum disease severity per the Myasthenia Gravis Foundation of America (MGFA) disease classification (5). Patients with positive anti-MUSK antibodies tend to have more pronounced bulbar symptoms and are less likely to respond to acetylcholine esterase inhibitors which can even worsen their symptomatology (3). In around $15 \%$ of patients with generalized $\mathrm{MG}$, no antibodies against AchR and MUSK are detected (2,7). These cases are identified as double-seronegative MG (dSN$\mathrm{MG}$ ). This subgroup of MG has clinical symptoms similar to MG with AchR antibodies in terms of muscle weakness distribution, severity of the disease and response to immunotherapy (1). This suggests that dSNMG is also an immune mediated dis- 
order that obviously affects the neuromuscular junction. Recently new antibodies that may be involved in MG have been discovered: anti-titin antibodies, anti Kv1.4 antibodies, antiRyR (ryanodine receptor) antibodies, anti cortactin antibodies (4). Anti-cortactin antibodies tend to appear in $20 \%$ of patients with dSNMG, but were also reported in $10 \%$ of patients with other autoimmune diseases (polymyositis) (1). Though so important for the diagnose, the test that measures these antibodies it is not yet available (1). In the near future, it is expected to be available as a routine test.

\section{CASE REPORT}

We report the case of a 21 years old previously healthy female that at 4 months after deliverance presented global muscle weakness, dysphonia, dysartria and dysphagia. The general physical exam was normal and the neurological exam revealed dysphagia, global lack of muscle strength, dysartria, dysphonia, no pyramidal syndrome, no diplopia. At admission: MG activity of daily living scale: $11 \mathrm{p}$ (M:24p), Myasthenic muscular score: 43p (M:100 p).

Biological tests revealed: anti acetylcholine receptor antibodies and anti MUSK antibodies - negative, TSH 4,68 $\mu \mathrm{IU} / \mathrm{ml}$ (normal range: 0,4-4 $\mu \mathrm{IU} /$ $\mathrm{ml}$ ), FT4 0,785 ng/dl (normal range: 0,89-1,76 ng/ dl), anti thyroglobulin antibodies $183,01 \mathrm{IU} / \mathrm{mL}$ (normal range: $<4,11 \mathrm{IU} / \mathrm{mL}$ ).

It was performed an EMG that concluded: Amplitudes of CAMP (compound action muscle potential) and NCS (nerve conduction stimulation) normal on right ulnar nerve and left facial nerve, with typical decremental responses at repetitive nerve stimulation testing on ADM (abductor digiti minimi) nerve and facial/nasalis nerve, and a chest CT which disclosed thymic hyperplasia in anterior mediastinum, non iodious (long axis $-5 \mathrm{~cm}$, short axis $-2 \mathrm{~cm}$ ).

It was established the diagnosis: double sero-negative myasthenia gravis Osserman stage IIb, autoimmune thyroiditis and Thymic hyperplasia.

The patient was treated with acetylcholine esterase inhibitors (pyridostigmine bromide) - $300 \mathrm{mg} /$ day, corticosteroids (prednisone) with progressive growth until $30 \mathrm{mg} /$ day and levothyroxine $25 \mu \mathrm{g} /$ day. The patient recovered almost completely and will be follwed up regularly. At discharge: $\mathrm{MG}$ activity of daily living scale: $2 p(\mathrm{M}: 24 \mathrm{p})$, myasthenic muscular score: 95p (M: 100p).

\section{DISCUSSION}

Myasthenia gravis is a rare autoimmune disorder (1/50,000 cases) (7). Double sero-negative myasthenia gravis appears in $15 \%$ of the patients diag-

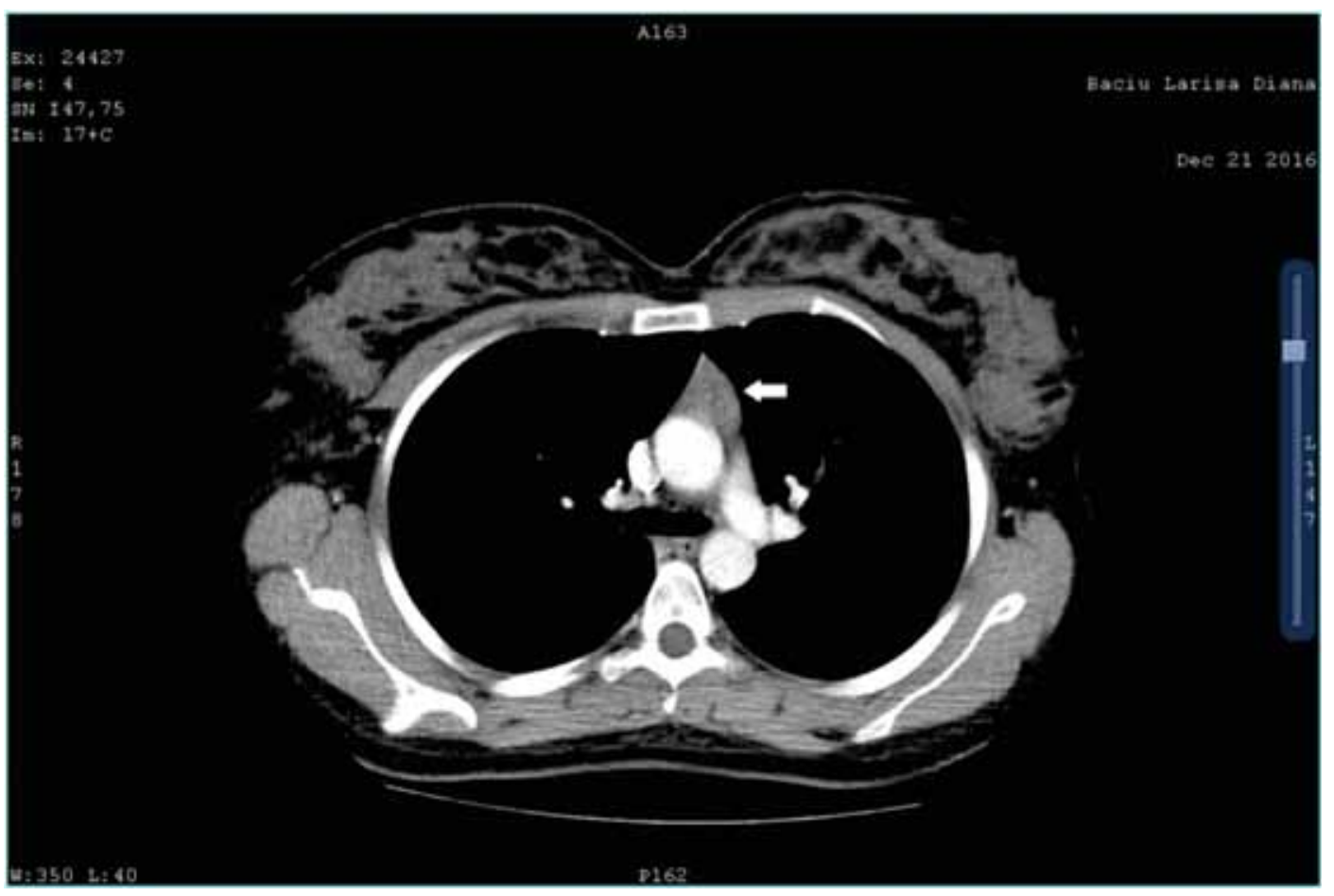

FIGURE 1.

Thoracic CT with contrast - thymic hyperplasia 
nosed with generalized MG $(7,8)$. Studies have shown that patients with MG and MUSK antibodies have a better response to prednisone and other immune therapies, including rituximab and a poor response to plasma exchange $(2,7)$. Currently, two antibodies are known to be specific for and play a causative role in MG: anti-AchR antibodies and antiMUSK antibodies but, among patients with MG, antibodies to potassium channels, agrin and the ryanodin receptor have been detected $(2,4)$. Patients with dSNMG should be tested for anticortactin antibodies too, but a test that could find these antibodies is not yet available (1). Some recent studies have concluded that the serological examination of antibodies and single-fiber electromyography confirm the diagnostic but they do not correlate with disease activity and response to treatment (5).

Myasthenia gravis it is diagnosed based on one or more of the following criteria:

1. positive results of AchR antibodies or antiMUSK antibodies;

2. electrophysiological findings that confirm a postsynaptic neuromuscular junction disorder and

3. positive therapeutic response to cholinesterase inhibitors (1). In dSNMG, the diagnosis is confirmed by abnormal findings on a neurophysiological exam and typical decremen- tal responses at repetitive nerve stimulation testing (1).

The association of another autoimmune disorders is very rare and appears in about $13-22 \%$ of the patients diagnosed with MG (9).

Thymic hyperplasia can worsen the disorder by initiating the immune response and producing antibodies, especially AchR antibodies (2). It is becoming clear that MG is not a single disease but a number of clinical subtypes that may be distinguished by their auto-antibody profile, as well as by thymic pathology and clinical presentation (2).

\section{CONCLUSIONS}

MG is a rare autoimmune disease that sometimes can be associated with other autoimmune disorders, most frequently autoimmune thyroiditis. It is specified in literature that in most cases, patients with thymic hyperplasia have anti-AchR antibodies. Our case was diagnosed with thymic hyperplasia but no antibodies were found in patient's plasma (dSNMG). When it is possible, the patient should be tested for anti-cortactin antibodies. This would not give a prognostic to the case, but it could help describing subtypes of MG related to the antibodies found in patient's plasma.

Conflict of interest: none declared Financial support: none declared

\section{REFERENCES}

1. Cortés-Vicente E., Gallardo E., Ángeles Martínez M., Díaz-Manera J. et al. Clinical Characteristics of Patients With Double-Seronegative Myasthenia Gravis and Antibodies to Cortactin, Jama Neurology, 2016;73(9):1099-104. doi: 10.1001/jamaneurol.2016.2032

2. Meriggioli M., Sanders D. Muscle autoantibodies in myasthenia gravis: beyond diagnosis? Expert Rev Clin Immunol. 2012 Jul; 8(5): 427-438. doi: 10.1586/eci.12.34

3. Meriggioli M., Sanders D. Autoimmune myasthenia gravis: emerging clinical and biological heterogeneity, Lancet Neurol. 2009 May; 8(5): 475-490. doi: 10.1016/S1474-4422(09)70063-8

4. Suzuki S., Utsugisawa K.,Nagane Y. Three Types of Striational Antibodies in Myasthenia Gravis, Autoimmune Diseases Volume 2011 (2011), Article ID 740583, 2011/740583

5. Gilhus N.E., Verschuuren J.J. Myasthenia gravis: subgroup classification and therapeutic strategies, Lancet Neurology, 2015; 14(10):1023-1036.

6. Rodríguez Cruz P.M., Al-Hajjar M., Huda S. et al. Clinical features and diagnostic usefulness of antibodies to clustered acetylcholine receptors in the diagnosis of seronegative myasthenia gravis, Jama Neurology, 2015; 72(6):642-649.

7. Daroff R.B., Fenichel G.M., Jankovic J. Bradley's Neurology in clinical practice, 2015

8. Conti-Fine B.M., Milani M., Kaminski H.J. Myasthenia gravis: past, present, and future, J Clin Invest, 2006; 116(11):2843-2854.

9. Nacu A., Andersen J.P., Lisnic V. et al. Complicating autoimmune diseases in myasthenia gravis: a review, published online 2015 doi: 1 0.3109/08916934.2015.1030614. 